ARTICLE

\title{
Qualitative indications for tracheostomy and chronic mechanical ventilation in patients with severe bronchopulmonary dysplasia
}

Sushmita Yallapragada (iD ${ }^{1 凶}$, Rashmin C. Savani ${ }^{1}{ }^{1}$, Sara Mūnoz-Blanco ${ }^{1}$, Joanne M. Lagatta (D) ${ }^{2}$, William E. Truog $^{3}$, Nicolas F. M. Porta (iD ${ }^{4}$, Leif D. Nelin ${ }^{5}$, Huayan Zhang (iD ${ }^{6}$, Shilpa Vyas-Read (D) ${ }^{7}$, Robert DiGeronimo (iD ${ }^{8}$, Girija Natarajan $^{9}$, Erica Wymore $^{10}$, Beth Haberman ${ }^{11}$, Joana Machry ${ }^{12}$, Karin Potoka ${ }^{13}$, Children's Hospital Neonatal Consortium severe bronchopulmonary dysplasia subgroup* and Karna Murthy ${ }^{4}$

(c) The Author(s), under exclusive licence to Springer Nature America, Inc. 2021

BACKGROUND: The decision to pursue chronic mechanical ventilation involves a complex mix of clinical and social considerations. Understanding the medical indications to pursue tracheostomy would reduce the ambiguity for both providers and families and facilitate focus on appropriate clinical goals.

OBJECTIVE: To describe potential indications to pursue tracheostomy and chronic mechanical ventilation in infants with severe BPD (sBPD).

STUDY DESIGN: We surveyed centers participating in the Children's Hospitals Neonatal Consortium to describe their approach to proceed with tracheostomy in infants with SBPD. We requested a single representative response per institution. Question types were fixed form and free text responses.

RESULTS: The response rate was high (31/34, 91\%). Tracheostomy was strongly considered when: airway malacia was present, $\mathrm{PCO}_{2} \geq 76-85 \mathrm{mmHg}, \mathrm{FiO}_{2} \geq 0.60, \mathrm{PEEP} \geq 9-11 \mathrm{~cm} \mathrm{H} 2 \mathrm{O}$, respiratory rate $\geq 61-70$ breaths/min, $\mathrm{PMA} \geq 44$ weeks, and weight $<10$ th $\%$ ile at 44 weeks PMA.

CONCLUSIONS: Understanding the range of indications utilized by high level NICUs around the country to pursue a tracheostomy in an infant with sBPD is one step toward standardizing consensus indications for tracheostomy in the future.

Journal of Perinatology (2021) 41:2651-2657; https://doi.org/10.1038/s41372-021-01165-9

\section{INTRODUCTION}

Infants with severe bronchopulmonary dysplasia (SBPD) have higher rates of cardiopulmonary and neurodevelopmental sequelae, growth failure, multiple medication exposures and the need for long-term ventilation that prompts a decision to perform a tracheostomy compared to preterm infants without sBPD [1-3]. Our prior study showed that although clinical variables were associated with and predictive of death or tracheostomy [4], there were wide (2-46\%) risk-adjusted rates of death or tracheostomy in 22 regional Neonatal Intensive Care Units (NICUs). This work raised questions about the variable and uncertain clinical indications to pursue tracheostomy and chronic mechanical ventilation in infants affected by sBPD.

Improved growth and developmental trajectory after tracheostomy and chronic mechanical ventilation are reported in infants with SBPD [5]. In addition, a recent study demonstrated that a tracheostomy, specifically, does not have negative effects on language or cognitive development in infants with sBPD [6]. For infants with chronic respiratory failure, establishing safe mechanical ventilation can begin a path to be discharged from the NICU [7]. However, this clinical path is associated with multiple risks including uncertain duration of the need for this support (often years), perioperative risks at the time of tracheostomy, serial procedures such as bronchoscopies, final decannulation with airway reconstruction, and the low, but life-threatening risk of accidental decannulation or obstruction at home [7, 8]. In addition, social considerations include the technological burden of maintaining a tracheostomy in areas of the country that lack home ventilation programs [9-11]. Home health provider shortages are a glaring problem for families with long-term medical needs. Children who are technology-dependent require lengthy hours of home health nursing. Limited availability of home health resources contributes to delays in care including family training, lack of equipment, and risk of readmission [9-11]. There is

\footnotetext{
${ }^{1}$ UT Southwestern Medical Center, Dallas, TX, USA. ${ }^{2}$ Medical College of Wisconsin, Milwaukee, WI, USA. ${ }^{3}$ Children's Mercy-Kansas City and the University of Missouri-Kansas City School of Medicine, Kansas City, MO, USA. ${ }^{4}$ Northwestern University \& Ann and Robert H. Lurie Children's Hospital of Chicago, Chicago, IL, USA. ${ }^{5}$ Nationwide Children's Hospital, Columbus, OH, USA. ${ }^{6}$ Children's Hospital of Philadelphia, Philadelphia, PA, USA. ${ }^{7}$ Emory University, Atlanta, GA, USA. ${ }^{8}$ Seattle Children's Hospital/University of Washington, Seattle, WA, USA. ${ }^{9}$ Children's Hospital of Michigan, Detroit, MI, USA. ${ }^{10}$ University of Colorado, Aurora, CO, USA. ${ }^{11}$ Cincinnati Children's Hospital Medical Center, Cincinnati, OH, USA.

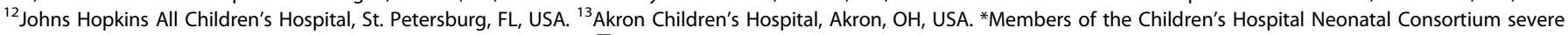
bronchopulmonary dysplasia subgroup are listed below Funding. ${ }^{凶}$ email: Sushmita.yallapragada@utsouthwestern.edu
}

Received: 6 March 2020 Revised: 13 May 2021 Accepted: 13 July 2021

Published online: 4 August 2021 
considerable regional variation. Rasooly et. al shows the probability of receiving home nursing across states ranged from 3.4 to $19.2 \%$, after adjusting for patient characteristics. Among those children who received home nursing the adjusted median days of care received ranged from 6.6 to 24.5 days [12].

Thus, the decision to pursue chronic mechanical ventilation involves a complex mix of clinical and social considerations [13]. Understanding the medical indications to pursue tracheostomy would reduce the ambiguity for both providers and families and facilitate focus on appropriate clinical goals.

Our objective was to identify provider-defined indications prompting the decision to pursue tracheostomy in infants with SBPD at regional NICUs in the Children's Hospitals Neonatal Consortium (CHNC). We hypothesized that although the indications for tracheostomy and chronic ventilatory support vary across institutions there are some indications common to a majority of institutions that could be used to inform eventual consensus guidelines.

\section{MATERIALS AND METHODS}

Participating hospitals in the CHNC were identified [14, 15]. Targeted responders were prospectively identified by direct contact: they included exclusively neonatology faculty from the 34 participating hospitals who were particularly invested in patients with SBPD at their institution and agreed to represent their group's practice regarding infants with sBPD. Each institution's targeted responder was surveyed using an online tool (RedCAP), and the survey was designed to receive one representative response per center. Responders were instructed to have group meetings and to respond based on their institutional, and not their individual, preferences. To account for variability amongst providers within an institution, we allowed free text and narrative responses to offer alternative approaches, ideas and thoughts. The authors were not blinded to each responder's institution affiliation.

The primary aim of this survey was to understand centers' indications for chronic ventilator support via tracheostomy. The secondary aim was to describe the prevalence of dedicated BPD clinical teams and their role in clinical decision making in the NICU for these patients.

The survey was presented in four sections with a total of 102 questions. The first section focused on patient inclusion/entry into BPD programs, if present, at each institution. The second section probed descriptive characteristics of the services provided to patients with SBPD at each institution. The third section requested clinical thresholds that prompted the decision for tracheostomy in affected infants. Factors that were specifically explored included respiratory support, interventions, and patients' clinical status: carbon dioxide thresholds $\left(\mathrm{PCO}_{2}\right)$, fraction of inspired oxygen $\left(\mathrm{FiO}_{2}\right)$, positive end expiratory pressure (PEEP), echocardiographic findings, and timing of tracheostomy. The final section contained open-ended questions that asked participants to describe clinical scenarios which may or may not prompt consideration of tracheostomy. These questions were:

1. Please describe a clinical situation wherein everyone at your institution would NOT decide to move forward with a tracheostomy in an infant with sBPD?

2. Please describe a clinical situation wherein everyone at your institution would decide to move forward with a tracheostomy in an infant with sBPD?

\section{This study was approved by the institutional review board at UT Southwestern}

The survey included questions that required dichotomous (Yes/No) responses, modified Likert scales, multiple choice, and narrative answers. The survey also included space for responders to elaborate and share institutional guidelines. Not every question was answered by each site, and responses occurred over a 9-month period in 2018. No compensation was provided for survey completion. Please see supplementary material for survey.

All replies were reviewed by the PI (SGY) and narrative responses were reviewed first by the $\mathrm{Pl}$, then by two co-authors (KM, JML) and a summary was presented to all authors to identify consistent themes. Reviewing authors were not blinded to the institution. Quotations selected for analysis were done by group consensus to illustrate conceptual themes (e.g., timing, severity of illness, co-morbidities) and to minimize repetition. Summative qualitative analysis was performed on open-ended responses to questions listed above, identifying codes, and calculating their frequency in order to identify patterns in the data and contextualize codes [16]. Descriptive results and qualitative analyses are reported.

\section{RESULTS}

Of the $34 \mathrm{CHNC}$ sites surveyed, 31 sites responded to the survey (91\%). Thirteen centers (42\%) had a self-identified BPD program. Among all centers, when asked about age at enrollment, the most common response $(10 / 13=77 \%)$ was for infants to be enrolled into the BPD program at 36 weeks post-menstrual age (PMA), and the second most common enrolled at 40-44 weeks PMA if infants still required respiratory support [17]. In addition, in descending frequency, time points for enrollment into BPD clinical programs were: At the time of tracheostomy, post-tracheostomy, survival without chronic ventilation or tracheostomy. No program enrolled infants prior to 32 weeks PMA into their BPD program. Centers reported the following disciplines were integral members of the sBPD team when making the decision for tracheostomy placement: respiratory therapy (45\%), nursing (39\%), advanced practice nurses (39\%), physical \& occupational rehabilitation (35\%), speech therapy (35\%), social work (35\%), pulmonary hypertension service $(32 \%)$, physiatry $(19 \%)$, pediatric critical care $(10 \%)$, and cardiology (10\%).

Centers were asked if there was an institution-specific consensus for the indication for tracheostomy for chronic ventilation that was used for patients with SBPD. Four centers (13\%) reported that they had such criteria and $26(87 \%)$ did not. The four centers who reported criteria did not elaborate further in the survey.

The specific indications for tracheostomy at each center, regardless of a dedicated BPD team, were then explored. There were no significant differences in responses between centers with and without a self-identified BPD team. Nearly all (93\%) of responders reported that infants with SBPD were commonly referred for respiratory failure. Most responders $(80 \%)$ noted that patients were specifically referred for tracheostomy, the decision having been strongly recommended or made at the referring hospital. The population of infants with SBPD least commonly referred to CHNC centers were those who already had a tracheostomy, and mainly referred for pulmonary management and transition to home (20\% of responders). Among 29 responders, the most common criteria that contributed to the decision for tracheostomy included: Airway and ventilation (total $32 \%)$, subcategorized as airway malacia (13\%), need for higher PEEP (8\%), and endotracheal positive pressure (11\%). Failure to thrive on noninvasive support (11\%), multiple courses of corticosteroid therapy (11\%), pulmonary hypertension (16\%), and poor growth and feeding (10\%) (Fig. 1). The least common indications for tracheostomy included: PMA (9\%), social considerations $(3 \%)$ and tolerance, avoidance or thriving with developmental care (8\%).

Table 1 describes the topics that were routinely considered when making the decision to recommend a tracheostomy with answers provided in a dichotomous manner. Findings on echocardiogram (right ventricular dilation, dysfunction, and hypertrophy) were important considerations $(60 \%, 80 \%, 50 \%$ respectively), among 30 responding centers. Systemic corticosteroid exposure was also an important consideration (69\%), among 29 responding centers. While growth parameters contributed to the decision, they were given a lower priority, including appropriate linear growth (41\%) and head growth (35\%), among 29 responding centers. Of note, none of the responders mentioned utilization of $\mathrm{CT}$, bronchoscopy and/or infant pulmonary function testing in their decision-making algorithm. 


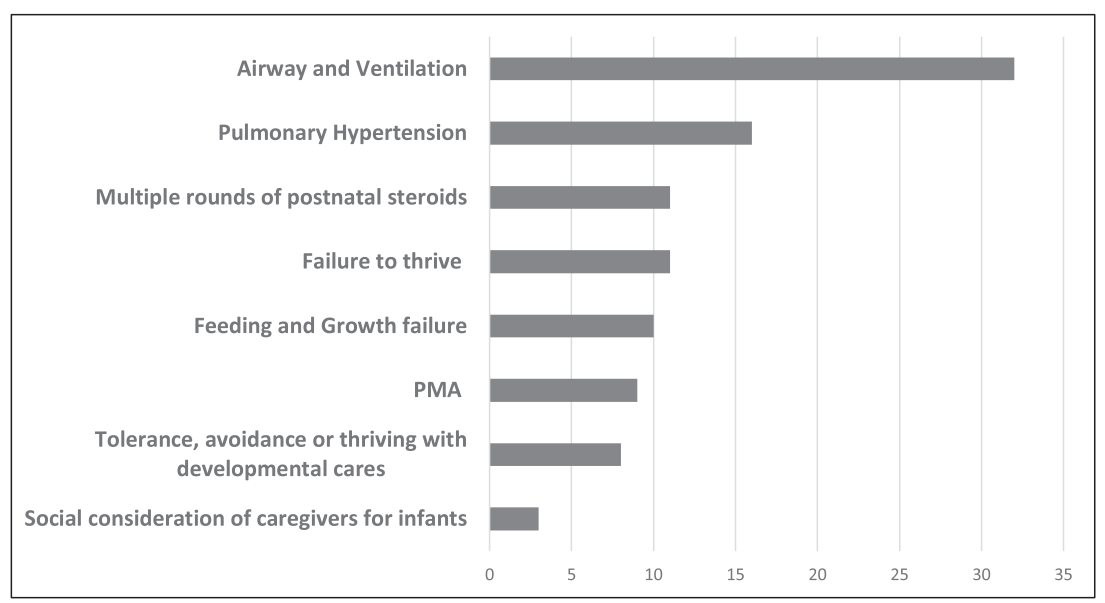

Fig. 1 Most common criteria for tracheostomy placement. Indications for tracheostomy, divided by reported level of importance (\%). $N=29$ responders.

Table 1. Parameters considered when making a decision for tracheostomy (Yes/No or N/A).

\begin{tabular}{|c|c|c|c|c|}
\hline Parameter \#centers, (\% total) & Yes & No & N/A & Notes \\
\hline $\begin{array}{l}\text { Appropriate } \\
\text { linear growth } \\
(n=29 \text { responders) }\end{array}$ & $12(41 \%)$ & $6(21 \%)$ & $12(41 \%)$ & $0.8-1 \mathrm{~cm} /$ week \\
\hline $\begin{array}{l}\text { Right ventricular (RV) hypertrophy on echo } \\
\text { ( } n=30 \text { responders) }\end{array}$ & $15(50 \%)$ & $10(33 \%)$ & $5(17 \%)$ & No additional information reported \\
\hline RV dilation on echo ( $n=30$ responders) & $18(60 \%)$ & $7(23 \%)$ & $5(17 \%)$ & No additional information reported \\
\hline $\begin{array}{l}\text { Systemic corticosteroid exposure } \\
\text { ( } n=29 \text { responders) }\end{array}$ & $20(69 \%)$ & $4(14 \%)$ & $5(17 \%)$ & Most reported $>2$ courses \\
\hline
\end{tabular}

Notes are written for most common ranges ( $>50 \%$ of hospitals) provided by institutions.

Responders used Likert scales to rate the level of importance of possible indications for tracheostomy and chronic ventilation (Table 2). Expressed as a percent of the total number of responding centers $(n=28)$ and reported in decreasing frequency, PMA $>44$ weeks' (82\%), the need for increased PEEP (62\%), pulmonary arterial hypertension (PAH) by echocardiography (55\%), multiple postnatal corticosteroid courses (52\%), multiple failed planned extubation attempts after 36 weeks' PMA (48\%), physiologic parameters $\left(\mathrm{PCO}_{2}\right.$ and $\left.\mathrm{F}_{\mathrm{i}} \mathrm{O}_{2}: 45 \%\right)$, failure to thrive with noninvasive support (45\%), projected concerns for abnormal future neurocognitive development $(45 \%)$, continued endotracheal positive pressure support (38\%), and social circumstances of the family (35\%) were the most prevalent indications cited by responders. Feeding intolerance was of lower importance in the decision to recommend tracheostomy (52\% minimal/none).

The next section surveyed the ranges of parameters that would prompt discussion of tracheostomy as an intervention, if all therapies affecting each parameter were optimized. Among 29 responders, the most common range for $\mathrm{PCO}_{2}$ was $76-85 \mathrm{mmHg}$, $\mathrm{F}_{\mathrm{i}} \mathrm{O}_{2}>0.60$, PEEP 9-11 cm H $\mathrm{H}_{2} \mathrm{O}$, respiratory rate $61-70$ breaths/min consistently, PMA $>44$ weeks, and weight $<10$ th percentile at 44 weeks PMA (Table 3).

Using qualitative analysis to analyze open-ended responses, we found that clinicians were generally less likely to agree on offering tracheostomy if the infant was demonstrating weaning of respiratory support, was on non-invasive ventilation (NIV), $\mathrm{FiO}_{2}$ lower than 0.4 , or PMA $<44$ weeks, even if on a ventilator or PMA up to 52 weeks if on NIV. Social considerations were only mentioned by four responders. Three were in the context of "the family does not want a tracheostomy"; one responder mentioned poor social support as a deciding factor. On the other hand, most clinicians seemed to agree that a tracheostomy is clearly indicated if the patient is of certain PMA and still intubated or has failed extubation. However, centers reported heterogeneity regarding which other clinical criteria, such as ventilator settings, use of corticosteroids, especially more than one course, were weighted and which combination. In addition, even when the same criteria were mentioned centers revealed heterogeneity; for example, some centers reported PMA of 44 weeks, others $>48$ weeks. The data suggests, however, that the decision to recommend a tracheostomy was generally agreed upon when PMA, ventilator, and medical therapies were exceeding what is "normal" (as defined by each center). Interestingly, none mentioned potential social or financial burdens on the affected families. A summary of our findings is available in Table 4. Sixteen centers responded. Dark rectangles represent each clinical criterion reported by the center. If the center was specific about the criterion, it is reported in white within the dark rectangles.

\section{DISCUSSION}

Severe BPD (sBPD) is a disease that primarily affects infants born $<32$ weeks' gestation, with a reported incidence of $42 \%$ among infants $501-750$ grams birth weight and 25\% among those with 751-1000 grams birth weight [18, 19]. Chronic mechanical ventilation with tracheostomy that serves to transition infants and their families from the NICU to the outpatient setting can be a viable management strategy but is associated with significant 
Table 2. Center reported ratings of the relative importance of indications in the decision to recommend tracheostomy.

\begin{tabular}{|c|c|c|c|c|}
\hline \multirow[t]{2}{*}{ Measure } & \multicolumn{4}{|l|}{ Importance } \\
\hline & Minimal/none; $n, \%$ & Moderate; $n, \%$ & High; $n, \%$ & Virtually always; $n, \%$ \\
\hline \multicolumn{5}{|l|}{ Airway and ventilation } \\
\hline Tolerance, avoidance or not thriving with developmental cares & $2(7 \%)$ & $8(27 \%)$ & $13(45 \%)$ & $6(21 \%)$ \\
\hline Multiple failed, planned extubation attempts $>36$ weeks' PMA & $2(7 \%)$ & $1(3 \%)$ & $14(48 \%)$ & $12(42 \%)$ \\
\hline $\begin{array}{l}\text { Multiple rounds of postnatal corticosteroid courses (defined } \\
>5 \text { days) to ameliorate the severity of BPD? }\end{array}$ & $2(7 \%)$ & $5(17 \%)$ & $15(52 \%)$ & $7(24 \%)$ \\
\hline Airway malacia & $1(3 \%)$ & 7 (24\%) & $15(52 \%)$ & $6(21 \%)$ \\
\hline Need for higher PEEP & $1(3 \%)$ & $8(28 \%)$ & $18(62 \%)$ & $2(7 \%)$ \\
\hline Post-menstrual age (PMA) & $1(3 \%)$ & $4(14 \%)$ & $12(41 \%)$ & $12(41 \%)$ \\
\hline \multicolumn{5}{|l|}{ Pulmonary arterial hypertension (PAH) } \\
\hline Growth (weight, height, head circumference) & $1(3 \%)$ & $13(45 \%)$ & $10(35 \%)$ & $5(17 \%)$ \\
\hline Failure to thrive on noninvasive support & $3(10 \%)$ & $5(17 \%)$ & $13(45 \%)$ & $8(28 \%)$ \\
\hline Feeding intolerance & $15(52 \%)$ & $9(31 \%)$ & $4(14 \%)$ & $1(3 \%)$ \\
\hline Social consideration of caregivers for infant & $6(20 \%)$ & $8(28 \%)$ & $10(35 \%)$ & $5(17 \%)$ \\
\hline
\end{tabular}
$N=28$ responders.

Table 3. Reported clinical indications for tracheostomy.

$\begin{array}{ll}\text { Parameter } & \text { Most commonly reported range } \\ \mathrm{PCO}_{2} & 76-85 \\ \mathrm{FiO}_{2} & >0.60 \\ \mathrm{PEEP} \text { requirement } & 9-11 \\ \text { Respiratory rate } & 61-70 \\ \mathrm{PMA} & >44 \text { weeks } \\ \text { Weight \% at PMA } & <10 \text { th } \\ N=29 \text { responders. } & \end{array}$

complications and morbidities including poor wound healing, air leak, stoma problems, accidental decannulation, and mucus plugs [20-22]. As infants grow older there are common reports of tracheal lesions such as fistulas, stenosis, collapse and peristomal granulation [23]. The decision to pursue tracheostomy and chronic home ventilation is often favored when the perceived alternatives are prolonged or uncertain duration of hospitalization, and/or mortality. This uncertainty of deciding to pursue tracheostomy is balanced against the potential hope that the infant will improve and will require a lower level of invasive support as well as decrease variation in institutional practices of decannulation $[24,25]$. Taking all of this into consideration, this study qualitatively captures the perceived indications that prompt the decision to pursue tracheostomy and describes the variability in both the selected criteria and their relative importance across multiple Level IV NICUs in North America.

Home chronic ventilation places a large burden on affected infants and families. This was first brought to the forefront of the American public by President Ronald Reagan in the early 1980s, followed by a funding waiver program established by the Department of Health and Human services, to assist families with ventilator dependent children at home $[26,27]$. The impact on families cannot be underscored enough as they deal with lifethreatening events felt to be related to equipment malfunction and/or tracheostomy occlusion, frequent re-hospitalizations and mortality $[13,28]$. In fact, through the course of this survey, we learned that many centers cite a $5-10 \%$ risk of adverse events after NICU discharge. Having a child that requires chronic home ventilation can burden caregivers, have significant effects on siblings and family relationships, restricts parental employment opportunities and adversely affects maternal health [29-33]. Families will spend a great deal of time on lengthy education to care for their child at home prior to discharge, and stable transition to appropriate home ventilator devices may be delayed significantly while awaiting home nursing care [28]. Often caregiver needs cannot be met with available home health nursing, even when approved by third-party payers. In 2016, The American Thoracic Society published guidelines regarding chronic home ventilation in pediatric patients $[34,35]$. In their recommendations, among other important items, they suggest an awake, trained caregiver be in the family's home at all times, at least two trained family caregivers prepared to care for the child at home, ongoing education for all caregivers, and updating and maintaining all durable medical equipment (DME) regularly. While these recommendations are necessary and prudent, the burden on the family as well as availability of home health care services is paramount [34].

PMA and specific physiologic parameters were the most common indications for tracheostomy. Responders also cited that airway malacia was an important parameter, followed by failure to thrive on noninvasive support, multiple courses of postnatal corticosteroids and endotracheal positive pressure. Though failure to thrive is subjectively determined and postnatal corticosteroids also have varied indications, our results suggest that a potential consensus definition of therapeutic failure would include $\mathrm{PCO}_{2}$ of $76-85, \mathrm{FiO}_{2}$ of $>0.60$, PEEP $9-11$, respiratory rate of $61-70, \mathrm{PMA}$ 


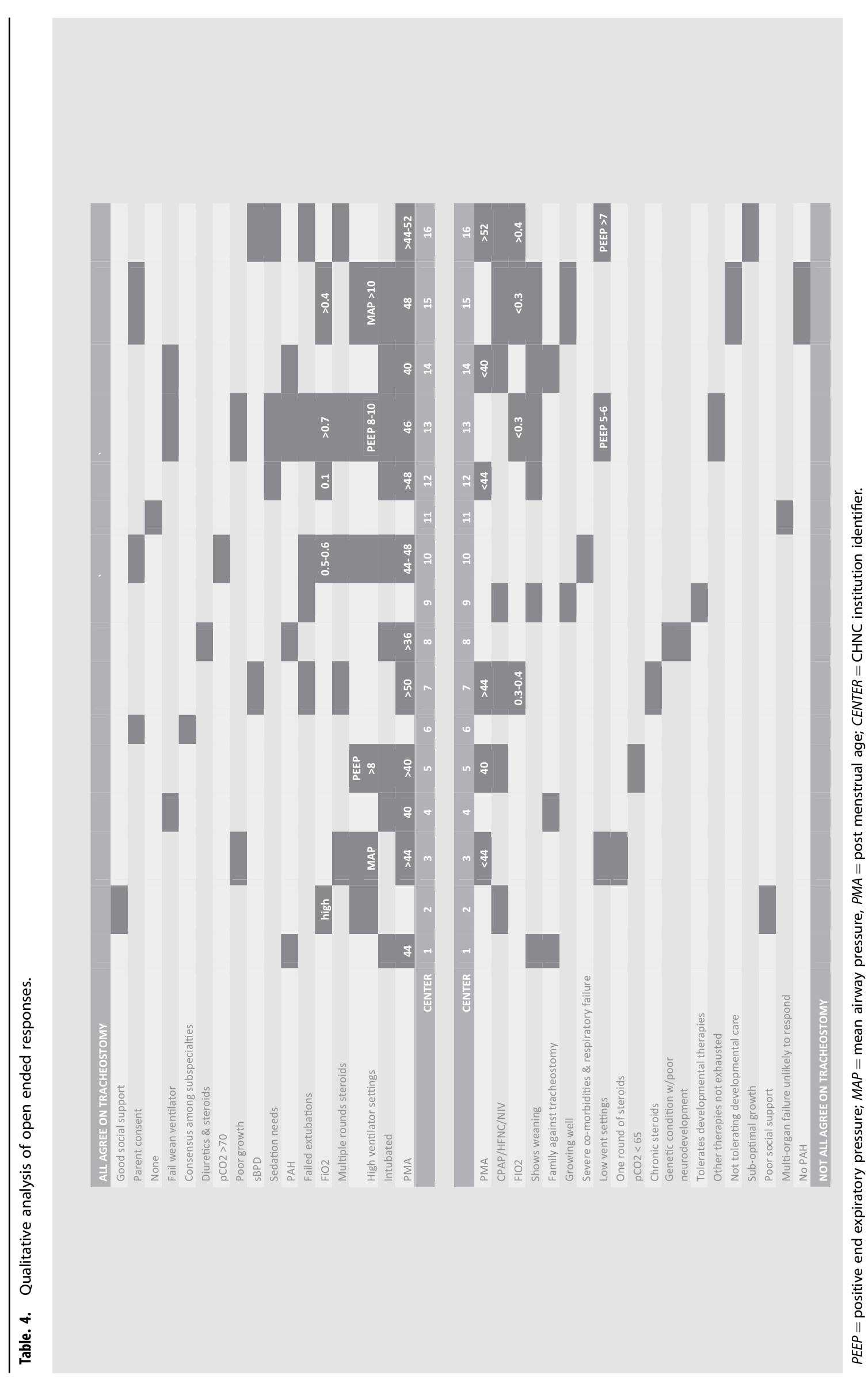

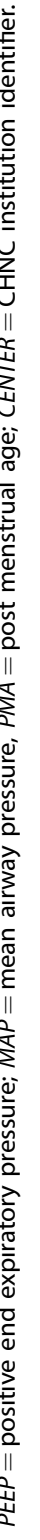


$>44$ weeks and weight $<10$ th percentile at this PMA. Most centers did agree that appropriate linear and head growth, corticosteroid exposure, and right heart insufficiency on echocardiogram were very important indications when considering tracheostomy. Although there was significant variability in approaches to tracheostomies across centers, it is interesting that there was some degree of agreement on which of the most severe scenarios would prompt consideration of tracheostomy.

The strengths of this survey include the high response rate (91\%) and the candid, detailed answers provided as one collective opinion per institution. On the other hand, although each responder replied on behalf of their institutional practice, this limited our ability to ascertain variability within the same institution. We included space for narration to allow responders to offer the nuances of their group's practices. Our survey may not have ideally delineated those infants receiving tracheostomy for airway obstruction, though in preterm infants with sBPD, lung disease was most likely the predominant respiratory issue. Further, infants at these NICUs are largely referral-based (>90\%) and biases that govern referral to these hospitals may not be generalizable to NICUs that care for mostly inborn populations. Referral timing, indications, and parental circumstances vary and, correspondingly, interventions to reduce the likelihood and/or severity of chronic respiratory failure are often instituted variably at referring hospitals.

In conclusion, our data demonstrate that reported indications for tracheostomy differ widely across regional NICUs. However, despite these differences between regional NICUs, there are indications that could allow the development of standardized indications in future studies. The development of standardized indications for tracheostomy and chronic ventilation techniques in infants with SBPD are needed to develop the evidence for best practices for the subset of infants with sBPD that require long-term respiratory support. Diagnostic certainty is needed to determine which infants require these interventions to achieve medical readiness for a safe, discharge home in a defined period of time, while achieving optimal long-term outcomes. Although clinical markers, genetic predisposition, assessments of the intrauterine environment, and biomarker validation hold promise to understand infants' prognoses more accurately, clearly the ability to provide evidence-based practices to these infants should also impact their long-term prognoses.

\section{REFERENCES}

1. Bamat NA, Kirpalani $H$, Feudtner $C$, Jensen $E$, Laughon $M$, Zhang $H$, et al. Medication use in infants with severe bronchopulmonary dysplasia admitted to United States children's hospitals. J Perinatol. 2019;39:1291-99.

2. Ehrenkranz RA, Walsh MC, Vohr BR, Jobe A, Wright L, Fanaroff A, et al. Validation of the National Institutes of Health consensus definition of bronchopulmonary dysplasia. Pediatrics. 2005;116:1353-60.

3. Jensen EA, Dysart K, Gantz MG, McDonald S, Barnat N, Keszler M, et al. The Diagnosis of Bronchopulmonary Dysplasia in Very Preterm Infants: an evidencebased approach. Am J Respir Crit Care Med. 2019;200:751-9.

4. Murthy K, Savani RC, Lagatta JM, Zaniletti I, Wadhawan R, Truog W, et al. Predicting death or tracheostomy placement in infants with severe bronchopulmonary dysplasia. J Perinatol. 2014;34:543-8.

5. Luo J, Shepard S, Nilan K, Wood A, Monk H, Jensen E, et al. Improved growth and developmental activity post tracheostomy in preterm infants with severe BPD. Pediatr Pulmonol. 2018;53:1237-44.

6. Cammack B, Noel-MacDonnell J, Cuna A, Manimtim W. Impact of tracheostomy on language and cognitive development in infants with severe bronchopulmonary dysplasia. J Perinatol. 2020;40:299-305.

7. Sobotka SA, Hird-McCorry LP, Goodman DM. Identification of Fail Points for Discharging Pediatric Patients With New Tracheostomy and Ventilator. Hosp Pediatr. 2016;6:552-7.

8. Berry JG, Goodman DM, Coller RJ, Agrawal R, Kuo D, Cohen E, et al. Association of Home Respiratory Equipment and Supply Use with Health Care Resource Utilization in Children. J Pediatr. 2019;207:169-75 e162.
9. Maynard R, Christensen E, Cady R, Jacob A, Ouellette Y, Podgorski H, et al. Home Health Care Availability and Discharge Delays in Children With Medical Complexity. Pediatrics. 2019;143:e20181951.

10. Wallis C, Paton JY, Beaton S, Jardine E. Children on long-term ventilatory support: 10 years of progress. Arch Dis Child. 2011;96:998-1002.

11. McDougall CM, Adderley RJ, Wensley DF, Seear MD. Long-term ventilation in children: longitudinal trends and outcomes. Arch Dis Child. 2013;98:660-5.

12. Rasooly IR, Shults J, Guevara JP, Feudtner C. State Variation in Posthospital Home Nursing for Commercially Insured Medically Complex Children. Pediatrics. 2020;146:e20192465.

13. Sobotka SA, Gaur DS, Goodman DM, Agrawal RK, Berry JG, Graham RJ. Pediatric patients with home mechanical ventilation: the health services landscape. Pediatr Pulmonol. 2019;54:40-6.

14. Murthy K, Dykes FD, Padula MA, Pallotto EK, Reber KM, Durand DJ, et al. The Children's Hospitals Neonatal Database: an overview of patient complexity, outcomes and variation in care. J Perinatol. 2014;34:582-6.

15. American Academy of Pediatrics Committee on Fetus And Newborn Levels of Neonatal Care: committee on Fetus and Newborn. Pediatrics. 2012;130:587-97.

16. Hsieh HF, Shannon SE. Three approaches to qualitative content analysis. Qual Health Res. 2005;15:1277-88.

17. Engle WA. American Academy of Pediatrics Committee on F, Newborn. Age terminology during the perinatal period. Pediatrics 2004;114:1362-4.

18. Natarajan G, Pappas A, Shankaran S, Kendrick D, Das A, Higgins R, et al. Outcomes of extremely low birth weight infants with bronchopulmonary dysplasia: impact of the physiologic definition. Early Hum Dev. 2012;88:509-15.

19. Bhandari A, Bhandari V. Pitfalls, problems, and progress in bronchopulmonary dysplasia. Pediatrics. 2009;123:1562-73.

20. Karkoutli AA, Brumund MR, Evans AK. Bronchopulmonary dysplasia requiring tracheostomy: a review of management and outcomes. Int J Pediatr Otorhinolaryngol. 2020;139:110449.

21. Klemm E, Nowak AK. Tracheotomy-Related Deaths. Dtsch Arztebl Int. 2017;114:273-9.

22. Watters K, O'Neill M, Zhu H, Graham RJ, Hall M, Berry J. Two-year mortality, complications, and healthcare use in children with medicaid following tracheostomy. Laryngoscope. 2016;126:2611-7.

23. Watters KF. Tracheostomy in Infants and Children. Respir Care. 2017;62:799-825.

24. Wirtz N, Tibesar RJ, Lander T, Sidman J. A Pediatric Decannulation Protocol: outcomes of a 10-Year Experience. Otolaryngol Head Neck Surg. 2016;154:731-4.

25. Henningfeld JK, Maletta K, Ren B, Richards KL, Wegner C, D'Andrea LA. Liberation from home mechanical ventilation and decannulation in children. Pediatr Pulmonol. 2016:51:838-49.

26. Treaster J. Cost President cite for Girl's Treatment is Described as 'Low'. New York Times, 1981. p. 25.

27. USDoHaH. Report of the Surgeon General's Workshop on Children with Handicaps and Their Families. Case Example: The Ventilator Dependent Child. United State Public Health Service. Division of Maternal and Child Health. 1983.

28. Sobotka SA, Foster C, Lynch E, Hird-McCorry L, Goodman DM. Attributable Delay of Discharge for Children with Long-Term Mechanical Ventilation. J Pediatr. 2019;212:166-71.

29. Quint RD, Chesterman E, Crain LS, Winkleby M, Boyce WT. Home care for ventilator-dependent children. Psychosocial impact on the family. Am J Dis Child. 1990;144:1238-41.

30. Patterson JM, Leonard BJ, Titus JC. Home care for medically fragile children: impact on family health and well-being. J Dev Behav Pediatr. 1992;13:248-55.

31. Petr CG, Murdock B, Chapin R. Home care for children dependent on medical technology: the family perspective. Soc Work Health Care. 1995;21:5-22.

32. Thyen U, Terres NM, Yazdgerdi SR, Perrin JM. Impact of long-term care of children assisted by technology on maternal health. J Dev Behav Pediatr. 1998;19:273-82.

33. Thyen U, Kuhlthau K, Perrin JM. Employment, child care, and mental health of mothers caring for children assisted by technology. Pediatrics. 1999;103:1235-42.

34. Sterni LM, Collaco JM, Baker CD, Carroll J, Sharma G, Brozek J, et al. An Official American Thoracic Society Clinical Practice Guideline: pediatric chronic home invasive ventilation. Am J Respir Crit Care Med. 2016;193:e16-35.

35. Moore PE, Boyer D, O'Connor MG, Baker C, Rettig J, Sterni L, et al. Pediatric Chronic Home Invasive Ventilation. Ann Am Thorac Soc. 2016;13:1170-2.

\section{AUTHOR CONTRIBUTIONS}

SY, RCS, JL, WT, NP and KM contributed to the design and implementation of the survey, as well as writing and reviewing the paper. SMB provided key qualitative research input and reviewed the paper. $L N, H Z$, SVR contributed to the design of the survey as well as editing the paper. $\mathrm{RD}, \mathrm{GN}, \mathrm{EW}, \mathrm{BH}, \mathrm{JM}, \mathrm{KP}$ contributed to writing and editing of this paper. 
FUNDING

None. RCS holds the William Buchanan Chair in Pediatrics at UT Southwestern.

\section{COMPETING INTERESTS}

Dr KM is Chair and Board Member of CHNC, a 501 (c)3 organization.

\section{ADDITIONAL INFORMATION}

Supplementary information The online version contains supplementary material available at https://doi.org/10.1038/s41372-021-01165-9.

\section{CHILDREN'S HOSPITAL NEONATAL CONSORTIUM SEVERE BRONCHOPULMONARY DYSPLASIA SUBGROUP}

Sushmita Yallapragada (iD ${ }^{1 凶}$, Rashmin C. Savani ${ }^{1}{ }^{1}$, Sara Mūnoz-Blanco ${ }^{1}$, Joanne M. Lagatta (iD ${ }^{2}$, William E. Truog ${ }^{3}$,

Nicolas F. M. Porta (iD) ${ }^{4}$, Leif D. Nelin ${ }^{5}$, Huayan Zhang (D) ${ }^{6}$, Shilpa Vyas-Read (D) ${ }^{7}$, Robert DiGeronimo (iD) ${ }^{8}$, Girija Natarajan $^{9}$, Erica Wymore ${ }^{10}$, Beth Haberman ${ }^{11}$, Joana Machry ${ }^{12}$, Karin Potoka ${ }^{13}$ and Karna Murthy ${ }^{4}$ 